Check for updates

Cite this: J. Mater. Chem. C, 2022, 10, 2194

Received 11th November 2021 Accepted 23rd December 2021

DOI: $10.1039 / d 1 t c 05452 j$

rsc.li/materials-c

\title{
Electronic and energy level engineering of directly fused porphyrin-conjugated polymers - impact of the central metal cation $\dagger$
}

\author{
Drialys Cardenas-Morcoso, (D) * Eloa Vey, Max Heiderscheid, Gilles Frache and \\ Nicolas D. Boscher (D) *
}

\begin{abstract}
The integration of porphyrins and their derivatives in functional devices for solar-assisted fuel production is both highly attractive and challenging due to the difficulties in processing them. This limitation is overcome in the gas-phase approach, particularly by oxidative chemical vapor deposition (oCVD), leading to the simultaneous synthesis and deposition of conjugated porphyrin coatings. We have investigated the impact of the metal cation of 5,15-diphenyl metalloporphyrins (MDPP; $M=\mathrm{Co}, \mathrm{Cu}, \mathrm{Mg}$, $\mathrm{Zn}, \mathrm{Pd}, \mathrm{Pt}, \mathrm{Ag}, \mathrm{Ru}, \mathrm{Ag}$, and $\mathrm{FeCl}$ ) on the dehydrogenative coupling reaction leading to fusedmetalloporphyrin thin films via OCVD and on the optoelectronic properties of the resulting thin films. We found that the nature of the chelated cation strongly affects the intermolecular coupling efficiency, as well as the occurrence of side reactions such as chlorination, intramolecular cyclization, demetallation/ re-metalation, and oxidation of the porphyrin core. Moreover, we discussed the influence of the abovementioned reactions on the optoelectronic properties of the fused metalloporphyrin coatings, in view of their potential application in photo-electrocatalytic systems. This study paves the way toward the engineering and future implementation of porphyrin-based systems for clean and efficient solar fuel production.
\end{abstract}

\section{Introduction}

Porphyrins and their derivatives play a fundamental role in essential natural processes - as chlorophyll in the photosynthesis driven by green plants - due to their outstanding light absorption and charge transport properties. Therefore, porphyrin-based materials are highly attractive in artificial light-harvesting systems, ${ }^{1,2}$ including photocatalytic and photoelectrochemical approaches towards hydrogen production from solar-assisted water splitting. ${ }^{3-5}$ The features of porphyrins (e.g. optoelectronic and catalytic properties) can be further enhanced by assembling them into large $\pi$-conjugated systems, e.g. porphyrin-based metal-organic frameworks (MOFs), ${ }^{6,7}$ bridged porphyrin conjugated polymers $(\mathrm{CPs})^{8}$ and directly fused porphyrin CPs. ${ }^{9,10}$ In contrast to inorganic semiconductors, even a small modification of the chemical structure of conducting and semiconducting coordination and covalent polymers can lead to a significant change of their optoelectronic properties and hence, the extension of their functional

Materials Research and Technology Department, Luxembourg Institute of Science and Technology, 28 avenue des Hauts-Fourneaux, Esch-sur-Alzette, Luxembourg. E-mail: drialys.cardenas@list.lu,nicolas.boscher@list.lu

$\dagger$ Electronic supplementary information (ESI) available. See DOI: 10.1039/d1tc05452j applications. ${ }^{2,6,11}$ In particular, the modification of the central metal ion and/or meso-substituents can alter the band gap and band edge positions of porphyrin-based $\mathrm{MOFs}^{6}$ and porphyrinbased CPs. ${ }^{8}$

In addition, the metal cation chelated at the center of the metalloporphyrin macrocycle - magnesium(II) in chlorophyll can act as a single-atom catalytic site, boosting the efficiency of a targeted chemical reaction. For example, cobalt(II)-bridged porphyrin CPs have been demonstrated as excellent earthabundant element heterogeneous catalysts for the oxygen evolution reaction (OER) ${ }^{12}$ from water oxidation and the oxygen reduction reaction (ORR).$^{13}$ When combining with zinc(II) porphyrins in a bridged porphyrin conjugated polymer, cobalt(II) porphyrins are also active for the hydrogen evolution reaction (HER) from water splitting. ${ }^{8}$ In this latter example, the periodically distributed type II-like heterojunction between adjacent zinc(II) and cobalt(II) porphyrins ensures the charge separation and transfer from zinc(II) porphyrins to cobalt(II) porphyrins. ${ }^{8}$ Directly fused porphyrin assemblies are also active catalysts. ${ }^{14,15}$ In particular, bimetallic copper(II)-fused porphyrin dimers were shown to be a highly active HER catalyst, demonstrating the direct fusion of the porphyrin macrocycles to be a promising strategy for enhanced electrocatalyst design. ${ }^{14}$ More recently, electrochemical and spectroscopic 




Scheme 1 OCVD reaction of a 5,15-diphenyl metalloporphyrin with iron(III) chloride as the oxidant, leading to triple, double, and single intermolecular coupling (green). Other possible side reactions are also represented: intramolecular coupling (blue), chlorination (red), a change in the oxidation state of the central metal cation (light blue), demetallation of the porphyrin core (violet), and further re-metalation with iron originating from the oxidant (orange).

investigations on fused iron(III) porphyrin complexes revealed their ability to store up to six electrons, added to the capability of the $\pi$-extended ligand to delocalize electrons across the multimetallic scaffold. ${ }^{16}$ These properties are particularly promising towards multielectron catalytic reactions driven by fused-metalloporphyrins. Despite these promising results, ${ }^{8,13,14}$ the difficult processability of porphyrins and particularly of any large $\pi$-conjugated porphyrin-based systems, owing to their non-solubility and non-meltability, jeopardizes their successful integration into functional devices.

Unlike wet chemistry methods, oxidative chemical vapor deposition (oCVD) enables the simultaneous synthesis and deposition of directly fused porphyrin CPs coatings in one step, without the need for solvents or post-treatment methods. ${ }^{17}$ In this process, a suitable volatile oxidant ${ }^{18}$ - usually iron(III) chloride - is used to promote the dehydrogenative coupling of porphyrins possessing free meso-positions, as represented in Scheme 1, yielding conductive thin films on a wide variety of substrates. ${ }^{17}$ As a suitable approach for the chemical engineering and molecular design of conjugated polymers, ${ }^{19}$ oCVD was used to improve the conductivity and functional properties of directly fused porphyrin-based CPs. Indeed, the mesosubstituents of porphyrins were reported to have a reduced impact on the polymerization of directly fused porphyrin CPs via oCVD,${ }^{20}$ in contrast to solution-based methods. ${ }^{21}$ However, aryl substituents bearing free ortho-positions were shown to undergo an intramolecular cyclization between the orthoposition of the aryl ring and the beta-position of the porphyrin macrocycle. Intramolecular cyclization is responsible for the molecular flattening of the directly fused porphyrin-conjugated polymer chains, which drastically affects their $\pi-\pi$ stacking facility and enhances the electronic properties of the films. ${ }^{20}$ Preliminary studies carried out by our group on the electro- and photocatalytic activity of directly fused copper(II) porphyrin conjugated polymer thin films for the heterogeneous HER showed that the phenyl substituent, enabling intramolecular cyclization, promotes superior catalytic performance over the mesityl substituent. ${ }^{15}$

Besides the dehydrogenative intermolecular coupling (polymerization) forming triple, double or single linkages between the porphyrin units and the dehydrogenative intramolecular coupling (cyclization), other side reactions can take place during the oCVD reaction of porphyrins. Namely, the chlorination of the macrocycle and the substituents, the demetallation and potential re-metalation of the porphyrin core, and oxidation of the central metal cation can take place (Scheme 1). ${ }^{22}$ The occurrence of several of these reactions, e.g. demetallation, remetalation and oxidation, is of course mainly influenced by the nature of the central metal cation. Additionally, it was shown that the central metal cation in 5,15-dimesityl porphyrins (MDMP; $\mathrm{M}=\mathrm{Ni}(\mathrm{II}), \mathrm{Cu}(\mathrm{II}), \mathrm{Zn}$ (II), $\mathrm{Co}(\mathrm{II}), \mathrm{Pd}(\mathrm{II})$, and $\mathrm{Fe}(\mathrm{III}) \mathrm{Cl}$ ) strongly affects the initial $\mathrm{C}-\mathrm{C}$ coupling step and consequently the formation of triply or doubly linked directly fused porphyrin tapes..$^{23}$ Therefore, the nature of the central metal cation is expected to have a double influence on the optoelectronic properties of the directly fused porphyrin CPs synthesized by oCVD.

With the aim of elucidating the direct and indirect (regioselectivity of the polymerization) influence of the central metal cation on the band gap and band edge positions of directly fused porphyrin-conjugated polymers, we studied the oCVD reaction of 5,15-diphenyl metalloporphyrins (MDPP; $\mathrm{M}=\mathrm{Co}$ (II), $\mathrm{Cu}(\mathrm{II}), \mathrm{Mg}$ (II), Zn(II), Pd(II), Pt(II), Ag(II), Ru(II), Ag(II), and Fe(III) $\mathrm{Cl}$ ). Phenyl was selected as the meso-substituent due to the superior catalytic ${ }^{15}$ and sensing properties ${ }^{24}$ of poly $(5,15-$ diphenyl metalloporphyrin) thin films over poly(5,15-dimesityl metalloporphyrin) ones. The occurrence of intermolecular coupling and other side reactions during OCVD was evidenced by ultraviolet-visible-near infrared (UV-Vis-NIR) spectroscopy, laser desorption ionization high-resolution mass spectrometry (LDI-HRMS), and X-ray photoelectron spectroscopy (XPS) analysis. The influence of the reactivity of 5,15-diphenyl metalloporphyrins on the electronic properties of the resulting coatings was evaluated using conductivity measurements by atomic force microscopy (c-AFM). Finally, from the combination of the optical and electronic characterization, we obtained the band alignment of the directly fused porphyrin CP thin films formed by oCVD, allowing their viability in solarassisted hydrogen production approaches to be assessed.

\section{Experimental}

\section{Reactants}

5,15-Diphenyl-metalloporphyrins chelated with different central metal cations $\left(\mathrm{CO}^{2+}, \mathrm{Cu}^{2+}, \mathrm{Mg}^{2+}, \mathrm{Zn}^{2+}, \mathrm{Pd}^{2+}, \mathrm{Pt}^{2+}, \mathrm{Ag}^{2+}, \mathrm{Ru}^{2+}\right.$, $\mathrm{Ag}^{2+}$, and $\mathrm{Fe}^{3+}-\mathrm{Cl}^{-}$) and the free-base $\left(\mathrm{H}_{2}\right)$ 5,15 diphenylporphyrin were obtained from PorphyChem $(98 \%)$ and were used without further purification. Based on previous reports, ${ }^{17,18}$ iron(III) chloride (97\%, Sigma-Aldrich) was chosen as the oxidising agent. Dichloromethane (HPLC grade $>99.8 \%$, SupraSolv ${ }^{\mathbb{R}}$ ) was used as the solvent for electrochemical and optical characterization. Tetrabutylammonium hexafluorophosphate $\left(\mathrm{TBAPF}_{6}\right.$, for electrochemical analysis, $\geq 99.0 \%$, Sigma-Aldrich) was used as the supporting electrolyte for the electrochemical measurements. 


\section{Porphyrin monomer characterization}

Thermogravimetric analyses (TGAs) of the porphyrin monomers were performed under an inert atmosphere (Ar) with a ramp of $10 \mathrm{~K}$ per minute. Cyclic voltammetry measurements were performed to estimate the redox potentials $\left(E_{1 / 2}\right)$ of the porphyrin monomers, in a three-electrode-cell and using an AUTOLAB potentiostat/galvanostat. A platinum working electrode, a platinum wire counter electrode, and a $0.01 \mathrm{M} \mathrm{Ag} /$ $\mathrm{AgNO}_{3}$ in acetonitrile reference electrode were used for the experiments. For each metalloporphyrin, cyclic voltammetry was performed using $1 \mathrm{mM}$ of the monomer in dichloromethane, containing $0.1 \mathrm{M} \mathrm{TBAPF}_{6}$ as the supporting electrolyte. The cyclic voltammograms were recorded at a $100 \mathrm{mV} \mathrm{s}^{-1}$ scan rate. Ferrocene was employed as the internal reference.

\section{Oxidative chemical vapor deposition (oCVD)}

The oCVD reaction was performed in a custom-built oCVD reactor (Scheme S1, ESI $\dagger$ ). Two crucibles were used to sublime the porphyrin and the oxidant, respectively. As previously reported, the different metal cations in the porphyrin core affect the sublimation temperature during the oCVD proces. ${ }^{23}$ Therefore, preliminary experiments were performed to determine the adequate sublimation temperature (from $230{ }^{\circ} \mathrm{C}$ to $290^{\circ} \mathrm{C}$ ) of each porphyrin monomer in order to sublime similar amounts of reactants, while keeping the porphyrin/oxidant ratio as constant as possible. Table S1 in the ESI $\dagger$ summarizes the deposition conditions used for each porphyrin investigated. On the other hand, the temperature of the oxidant's crucible was always kept at $170{ }^{\circ} \mathrm{C}$. Glass microscope slides (MenzelGläser Superfrost $\left.{ }^{\mathbb{R}}\right)$, bare and platinum-coated silicon wafers (Siegert Wafer ${ }^{\circledR}$ ) and interdigitated chips (OFET Gen4, Fraunhofer) were used as substrates. Prior to deposition, all the substrates were cleaned with absolute ethanol (99.98\%, VWR chemicals $\left.{ }^{\mathbb{R}}\right)$ and dried with nitrogen gas. The substrate holder was kept at $150{ }^{\circ} \mathrm{C}$ for all the depositions. The pressure inside the oCVD reactor was kept at $10^{-3}$ mbar, under an argon (99.999\%, Air Liquide) atmosphere. The deposition time was set to 30 minutes for all the experiments. Additionally, reference porphyrin monomers coatings were obtained under the same conditions, without supplying the oxidant.

\section{Film characterization}

The ultraviolet-visible-near infrared (UV-Vis-NIR) spectra of the thin films deposited on glass slides were recorded with a PerkinElmer Lambda 1050 spectrometer, in the transmission (T) mode, and at a wavelength interval of 300-2500 $\mathrm{nm}$. The absorbance $(A)$ was calculated as: $A=-\log (T)$. From the absorbance spectra, the direct optical band gap of the fusedmetalloporphyrins was estimated through the Tauc plot as: $(\alpha h \nu)^{1 / n}=A\left(h \nu-E_{\mathrm{g}}\right)$, where $\alpha$ is the absorbance coefficient, $n=1 / 2$ for direct transitions, $h$ is the Planck's constant and $\nu$ is the wavelength number. The absorbance coefficient was calculated as: $\alpha=\ln (10) A / l$, where $l$ is the film's thickness. The film's thickness was determined with an alpha-SE spectroscopic ellipsometer. The spectroscopic ellipsometry measurements were acquired at three angles $\left(65^{\circ}, 70^{\circ}\right.$ and $\left.75^{\circ}\right)$, in the wavelength range of 500-900 $\mathrm{nm}$. The data were fitted to a Cauchy model. The films were rinsed with dichloromethane to record the absorbance spectra of the DCM-soluble phase in transparent quartz cuvettes of $3.5 \mathrm{~mL}$ capacity and $1 \mathrm{~cm}$ of light path. The rinsed glass slides were also analyzed for comparison with the as-deposited films.

Scanning electron microscopy (SEM) images were recorded using a FEI Quanta 200F system. X-ray photoelectron spectroscopy (XPS) measurements were performed with a Kratos Axis Ultra DLD, instrument using a monochromatic Al K $\alpha$ X-ray source of energy of $1486.6 \mathrm{eV}$, at a power of $105 \mathrm{~W}$. Charge calibration was accomplished by fixing the binding energy of carbon (C 1s) to $285.0 \mathrm{eV}$.

Laser desorption/ionization high-resolution mass spectrometry (LDI-HRMS) measurements were performed on an APMALDI UHR ion source (MassTech, Inc.) coupled to an LTQ/ Orbitrap Elite (ThermoScientific) system. In-source fragmentation $(E=70 \mathrm{~V})$ was used to prevent the formation of clusters. The measurements were performed on a Si wafer coated with the directly fused porphyrin CP films, placed directly on the sample holder. LDI-HRMS measurements and analysis of metalloporphyrin coatings have been described in detail elsewhere. ${ }^{18,22,23}$

The conductivity of the oCVD coatings was acquired using the c-AFM mode of an Innova AFM (Bruker) system. Conductive AFM tips, ElectriMulti75-G from BudgetSensors coated with a layer of $5 \mathrm{~nm}$ chromium and $25 \mathrm{~nm}$ of platinum with nominal spring constants of $3 \mathrm{~N} \mathrm{~m}^{-1}$ and nominal radius $<25 \mathrm{~nm}$ were used. The samples were deposited on monocrystalline silicon wafers coated with platinum and connected to the conductive stage via a silver colloidal ink. A bias of $+4 \mathrm{~V}$ was applied to the back electrode of the samples, while the grounded conductive tip collected electrons for the current measured using an amplifier (DLPCA-200, Femto). A $109 \mathrm{~V} \mathrm{~A}^{-1}$ amplification was used for the pA current range, while a $107 \mathrm{~V} \mathrm{~A}^{-1}$ was used for those ones in the nA range.

\section{Results and discussion}

\section{Reactivity of diphenyl-substituted metalloporphyrins in oCVD}

Prior to the oCVD processing, the redox potential of the metalloporphyrin monomers was determined through cyclic voltammetry measurements. Fig. S1 of the ESI $\uparrow$ show the cyclic voltammograms of the metalloporphyrin monomers. The redox potentials $\left(E_{1 / 2}\right)$ of ZnDPP, CuDPP, CoDPP, MgDPP, PtDPP, AgDPP and RuDPP monomers were estimated at the midpoint potentials for the reversible and quasi-reversible waves, as the average of the anodic and cathodic peak potentials. The cyclic voltammetry of NiDPP, PdDPP, FeDPP, and $\mathrm{H}_{2}$ DPP show irreversible redox couples. For these samples, the $E_{1 / 2}$ was estimated as the half-potential peak of the voltammograms, corresponding to the potential at half the maximum current in the cyclic voltammograms. ${ }^{25}$ All the values are summarized in Table 1. The obtained values are consistent with those 
Table 1 Redox potential $\left(E_{1 / 2}\right)$ of di-phenyl substituted metalloporphyrins, obtained from cyclic voltammetry measurements of the porphyrin monomers

\begin{tabular}{llll}
\hline Porphyrin & $E_{1 / 2}\left(\mathrm{~V} v s . \mathrm{Fc} / \mathrm{Fc}^{+}\right)$ & Porphyrin & $E_{1 / 2}\left(\mathrm{~V} v s . \mathrm{Fc} / \mathrm{Fc}^{+}\right)$ \\
\hline MgDPP & 0.24 & AgDPP & 0.55 \\
CoDPP & 0.27 & $\mathrm{H}_{2} \mathrm{DPP}$ & 0.56 \\
ZnDPP & 0.41 & PdDPP & 0.60 \\
RuDPP & 0.42 & PtDPP & 0.64 \\
CuDPP & 0.54 & FeClDPP & 0.69 \\
NiDPP & 0.54 & &
\end{tabular}

previously reported for di-mesityl substituted metalloporphyrins. ${ }^{23}$ All the di-phenyl substituted metalloporphyrins have $E_{1 / 2}$ values lower than the oxidation potential of the $\mathrm{Fe}^{3+}$ / $\mathrm{Fe}^{2+}$ couple $(0.77 \mathrm{~V})$, supporting the selection of an iron(III)based oxidant for the dehydrogenative coupling of the selected metalloporphyrins.

It is worth mentioning that after the oCVD reaction, the redox potential of the resulting fused-metalloporphyrins can significantly vary. As previously reported by Moore and coworkers for copper and iron-based bi-nuclear fused porphyrins, such a difference of redox events between the monomer and the fused porphyrins is attributed to the enhanced electronic delocalization resulting from the extended aromaticity of the fused architecture. ${ }^{14,16}$

To set an adequate sublimation temperature of the selected metalloporphyrins during the oCVD reaction, their thermal stability was assessed by thermogravimetry (Fig. S2 in the ESI $\dagger$ ). All the di-phenyl substituted metalloporphyrins investigated in this work are thermally stable up to at least $300{ }^{\circ} \mathrm{C}$ confirming their suitability for oCVD processing. Two series of thin films were prepared from (i) the oCVD reaction of the metalloporphyrins with iron(III) chloride (denoted as PMDPP) and (ii) from the sublimation of the metalloporphyrins (denoted as SMDPP). The sublimation temperatures used for the oCVD reaction of each metalloporphyrin investigated are summarized in the Table S1 of the ESI, $\dagger$ including the amount of porphyrins and oxidant consumed during the thin film deposition experiments.

The SEM analysis of the films prepared by OCVD shows homogeneous coverage and a relatively smooth and dense surface, as depicted in Fig. S3 of the ESI, $\dagger$ in agreement with previous reports. ${ }^{20,26}$ The particles and islands disseminated across the surfaces of the oCVD films are attributed to the inclusions of both the unreacted oxidant and oxidant byproducts. ${ }^{22}$

Most of the oCVD coatings exhibit a notable color difference compared to the reference coatings obtained from the sublimation of the metalloporphyrin monomer without any oxidant, as shown in Fig. 1a. Such color changes are indicative of the occurrence of some of the reactions depicted in Scheme 1, including the formation of directly fused porphyrin tapes. ${ }^{17,18}$ In particular, the oCVD coatings obtained from $\mathrm{Zn}, \mathrm{Cu}, \mathrm{Co}, \mathrm{Mg}$, Ni and Pt di-phenyl substituted porphyrins (pZnDPP, pCuDPP, pCoDPP, pMgDPP, pNiDPP and PtDPP, respectively) exhibit an intense green coloration, different from the orange of the (a)


Fig. 1 (a) Digital pictures of the as-deposited OCVD coatings from 5,15diphenyl metalloporphyrins with different central metal cations, in the presence of iron(II) chloride (top row). The films from the sublimed monomers without an oxidant are shown as references (bottom row). (b) and (c) UV-Vis-NIR spectra of the as-deposited oCVD coatings. For better comparison, the absorbance spectra are normalized to their Soret bands.

sublimed reference thin films. This is consistent with previous studies on the oCVD reactivity of porphyrins hinting at the retention of the porphyrin macrocycle upon sublimation, and their oxidative dehydrogenation and polymerization in the presence of a suitable oxidant. ${ }^{18}$ Conversely, thin films from the oCVD reaction of the $\mathrm{Pd}, \mathrm{Ag}, \mathrm{Ru}, \mathrm{Fe}$ and free-base porphyrins (pPdDPP, pAgDPP, pRuDPP, pFeDPP, and $\mathbf{p} \mathbf{H}_{2} \mathbf{D P P}$, respectively) exhibit yellow and reddish colorations, that in some cases do not strongly differ from their respective reference thin films (e.g. sRuDPP and pRuDPP), as depicted in Fig. 1a, suggesting less efficient coupling reaction.

Further insights into the oCVD reaction efficiency toward directly fused porphyrin CPs are revealed from the UV-Vis-NIR spectra of the as-prepared thin films. Broadened Soret and Q bands, and absorption in the NIR spectral region were observed for PZnDPP, pCuDPP, pCoDPP, pMgDPP, pNiDPP, pPtDPP, and pRuDPP thin films, as depicted in Fig. $1 \mathrm{~b}$ and c. This enhanced absorption is indicative of the formation of multiply fused porphyrin tapes, due to the intensified and red-shifted Soret and Q-bands deep in the NIR region.

In particular, the pZnDPP, pCuDPP, pCoDPP, pMgDPP, pNiDPP, and pPtDPP films exhibit appreciable absorption above $1500 \mathrm{~nm}$, pointing to the significant formation of triply linked porphyrin tapes. The low redox potentials of ZnDPP, CoDPP, and MgDPP explain the highest degree of fusing derived from these monomers, as suggested by the UV-VisNIR spectra. However, the higher redox potentials of PtDPP and NiDPP, coupled with the high degree of fusing of pPtDPP and pNiDPP indicated by the UV-Vis-NIR spectra, suggest more complex relationships in the successful dehydrogenative coupling of metalloporphyrins in oCVD.

On the other hand, the pPdDPP and pAgDPP films are essentially transparent above $1500 \mathrm{~nm}$, as depicted in Fig. 1c, suggesting the formation of mainly doubly linked porphyrin 
tapes. Moreover, the absorbance spectra obtained for pFeDPP and $\mathbf{p} \mathbf{H}_{2}$ DPP films are rather similar to those of their respective reference sublimed films, displaying neither significant shift of the Soret and Q-bands, nor absorption in the NIR region, as shown in Fig. S4 of the ESI. $\dagger$ Such observations confirm that these porphyrins failed to yield directly fused porphyrin tapes, or at least with a lower efficiency.

The analysis of both the thin films after rinsing with a nonpolar solvent, i.e. dichloromethane (DCM), and the collected DCM-soluble phase provides significant insights into the formation of directly fused porphyrin chains and other modifications occurring during the oCVD reaction. As shown in the digital picture of the films (Fig. S5, ESI $\dagger$ ) the pZnDPP, pCuDPP, pCoDPP, pMgDPP, pNiDPP, and pPtDPP coatings are almost insoluble in dichloromethane, suggesting the formation of highly fused porphyrin oligomers. The UV-Vis-NIR spectra of these films after DCM rinsing remain superimposable with those of the as-deposited films, as depicted in Fig. S4(a)-(f), $\mathrm{ESI}, \dagger$ while there is a clear absence of NIR absorption in the DCM-soluble fractions of these films (Fig. S6(a)-(f), ESI $\dagger$ ) pointing to the retention of the multiply fused porphyrin tapes in the films. Conversely, the pPdDPP, pAgDPP, pRuDPP, pFeDPP, and $\mathbf{p H}_{2}$ DPP oCVD coatings are visibly partially dissolved in dichloromethane (Fig. S5, ESI $\dagger$ ), also confirmed from the UV-Vis-NIR spectra of the oCVD films after rinsing, compared to the as-deposited oCVD films as shown in Fig. S4(g)-(k) (ESI $\dagger$ ). The rinsing experiment using DCM was also performed in the reference sublimed samples (in the absence of the oxidant), for comparison. In all cases, the sublimed films were fully dissolved, as expected from the solubility of the porphyrin monomers in non-polar solvents such as DCM, confirmed by the UV-VIS-NIR analysis of the DCM-soluble fraction (Fig. S6, $\mathrm{ESI} \dagger)$.

To reach a deeper understanding on the reactivity of diphenyl substituted metalloporphyrins in oCVD, laser desorption/ ionization high-resolution mass spectrometry (LDI-HRMS) and X-ray photoelectron spectroscopy (XPS) analyses were performed on both the sublimed references and oCVD films. It is worth mentioning that the LDI-HRMS spectra do not provide a complete representation of all the oligomer chains formed, since the instrument only detects $\mathrm{m} / \mathrm{z}$ values lower than 4000 . In addition, long and highly fused porphyrins are difficult to ionize, hindering the detection of larger oligomers. Yet, the LDI-HRMS analysis of the prepared films, in combination with UV-Vis-NIR and XPS analyses, provides insightful information into the reactivity and conformations of directly fused metalloporphyrins.

Irrespective of the central metal cation, the distribution of peaks corresponding to the presence of monomers $(n=1)$ and oligomers $(n>1)$ is seen in the LDI-HRMS spectra of all the oCVD films, as depicted in Fig. S7(a)-(k) of the ESI. $\dagger$ Structures up to pentamers are notably detected, as representatively shown in Fig. 2a corresponding to the pCuDPP oCVD films, indicating the successful fusing of the porphyrin units by dehydrogenative $\mathrm{C}-\mathrm{C}$ coupling. Furthermore, the chlorination side reaction is clearly present in all the oCVD coatings giving

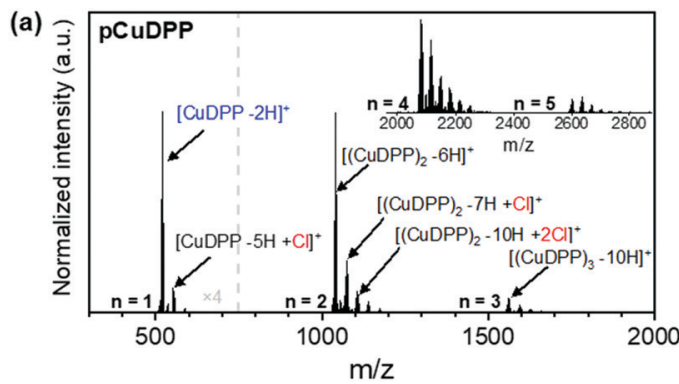

(b)



Fig. 2 (a) LDI-HRMS spectrum of the oCVD film prepared from CuDPP, in the 400-3000 $\mathrm{m} / \mathrm{z}$ range, showing the oligomer formation due to dehydrogenative $\mathrm{C}-\mathrm{C}$ coupling ( $n=$ degree of oligomerization) and the occurrence of chlorination side reaction. (b) LDI-HRMS spectrum in the dimer region of pCuDPP (red) in comparison with the simulated spectra of the dimers $\left[(\mathrm{CuDPP})_{2}-(\mathrm{H} 2)_{n}\right]^{+}$(n comprising between 1 and 5$)$.

rise to peak distributions that shifted from $35 \mathrm{~m} / \mathrm{z}$ corresponding to the exchange of hydrogen atoms by chlorine atoms (Fig. 2a). We should note that, unlike previously studied dimesityl substituted porphyrins, where meso-mesityl substituents with blocked ortho positions were used, diphenyl substituted porphyrins are highly prone to intermolecular cyclization. This effect is shown in Fig. 2b where both intermolecular (polymerization) and intramolecular (cyclization) dehydrogenative coupling (Scheme 1) occur to form $\left[(\mathrm{CuDPP})_{2}-\left(\mathrm{H}_{2}\right)_{n}\right]^{+}$ dimers in which more than three hydrogen pairs (up to three intermolecular $\mathrm{C}-\mathrm{C}$ bonds between two porphyrins) are eliminated. Indeed, significant contributions of the $\left[(\mathrm{CuDPP})_{2}-8 \mathrm{H}\right]^{+}$ and $\left[(\mathrm{CuDPP})_{2}-10 \mathrm{H}\right]^{+}$species to the experimental signal were observed, pointing to the formation of a minimum of one and two intramolecular $\mathrm{C}-\mathrm{C}$ coupling of triply fused dimers, respectively.

According to their respective UV-Vis-NIR spectra that suggest the formation of highly fused porphyrin tapes (Fig. 1b), the LDIHRMS spectra of the pCoDPP, pNiDPP, and pPtDPP oCVD films are dominated by peaks related to altered monomers and oligomers $\left[(\mathrm{MDPP})_{n}-\left(\mathrm{H}_{2}\right)_{m}\right]^{+}(m \geq n)$ in which multiple hydrogen pairs are eliminated. These observations are in good 
agreement with the formation of double and triple linkages between the porphyrin units chelated with $\mathrm{Cu}$ (II), $\mathrm{Co}$ (II), and $\mathrm{Ni}$ (II) cations, as previously reported for the oCVD reaction of 5,15-dimesityl-metalloporpyrins (MDMPs), ${ }^{20}$ being ascribed to the mixed $a_{1 \mathrm{u}} / a_{2 \mathrm{u}}$ molecular symmetry of their radical cations. Furthermore, besides the intramolecular cyclization enabled by the free-ortho positions of the phenyl substituent and the chlorination induced using iron(III) chloride, no other side reactions were detected for PCUDPP, pNiDPP, and pPtDPP. In the case of pCoDPP, a change in the oxidation state of the central metal cation $\mathrm{Co}^{2+}$ was detected from XPS analysis (Fig. S8a, ESI $\dagger$ ). Precisely, the XPS Co $2 p$ core level peak is broadened and shifted $1.1 \mathrm{eV}$ towards higher binding energies compared to the sublimed porphyrin (SCoDPP) where only $\mathrm{Co}^{2+}$ is present. This is indicative of the presence of both $\mathrm{Co}^{2+}$ and $\mathrm{Co}^{3+}$ oxidation states in the directly fused porphyrin CPS formed from the oCVD reaction of CoDPP. This observation is supported by a similar shift observed in the XPS N 1s core level region, depicted in Fig. S8b (ESI $\dagger$ ). These phenomena of cobalt-based porphyrins have already been evidenced in the literature. ${ }^{23,27,28}$ It is worth mentioning that the analysis of the XPS spectra of Pd 3d, Ni 2p, Ru 3d, Cu 2p, and Pt 4f core levels of the reference and oCVD thin films (Fig. S9a-f, ESI $\dagger$ ) suggests no alterations of these central metal cations.

UV-Vis-NIR analysis also suggested an efficient intermolecular dehydrogenative coupling reaction for the pZnDPP and pMgDPP coatings, fostered by the low redox potentials of the respective monomers (Table 1 ). However, although the signals corresponding to the formation of doubly and triply fused oligomers were detected and further confirmed by comparison with the simulated isotopic patterns (Fig. S10 of ESI $\dagger$ ), a pronounced demetallation of the porphyrin cores is observed in the LDI-HRMS spectra (Fig. 3a and c). A more remarkable effect is observed for pMgDPP whose LDI-HRMS spectrum is dominated by the peaks related to fused free-base porphyrin dimers $\left[\left(\mathrm{H}_{2} \mathrm{DPP}\right)_{2}-6 \mathrm{H}\right]^{+}$. Demetallation is also evidenced by the XPS analysis of the oCVD films. The $\mathrm{N} 1 \mathrm{~s}$ region of a free-base porphyrin is characterized by the presence of two signals at 400.0 eV and $397.9 \mathrm{eV}$ binding energies, characteristics of amino- and imine-pyrrole nitrogen environments. As shown in Fig. 3b and d, the pZnDPP and pMgDPP films displayed signals at $398.5 \mathrm{eV}$ and $398.4 \mathrm{eV}$, respectively, corresponding to nitrogen in its initial $\mathrm{Zn}^{2+}$ and $\mathrm{Mg}^{2+}$ chemical environments. As shown in Fig. $3 b$ and $d$, the pZnDPP and pMgDPP films (a)



(c)

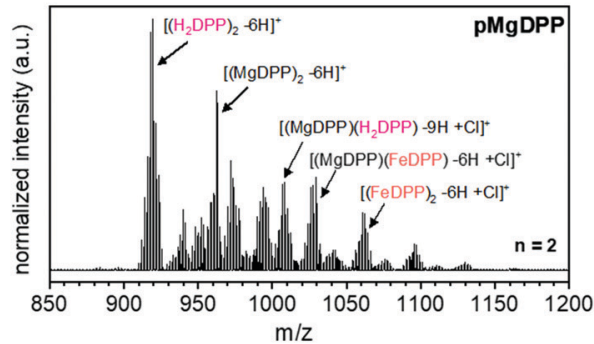

(e)

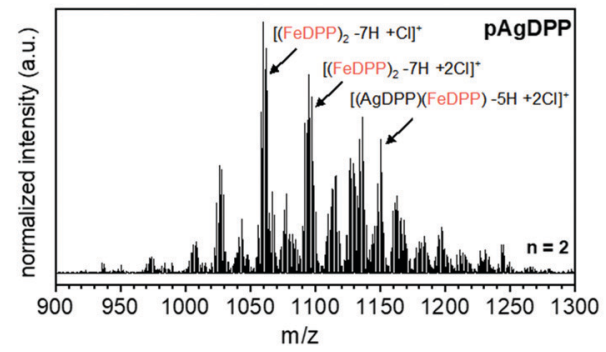

(b)

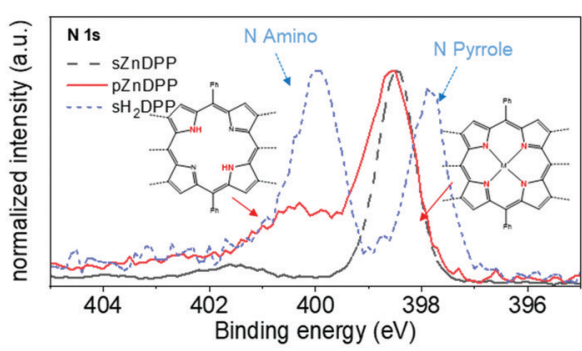

(d)

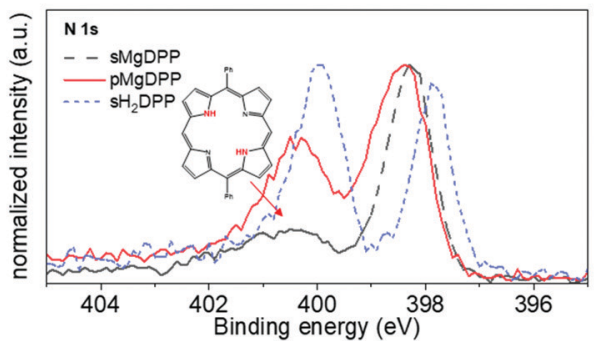

(f)

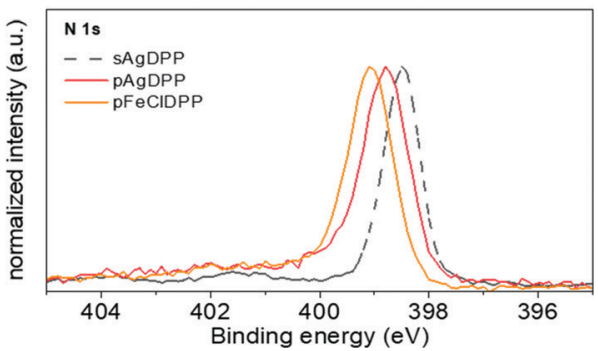

Fig. 3 LDI-HRMS spectra in the dimer regions $(n=2)$ of the oCVD films prepared from: (a) ZnDPP, (c) MgDPP and (e) AgDPP. XPS spectra of the N 1s regions of (b) sublimed reference and OCVD films prepared from ZnDPP. The sublimed free-base porphyrin spectrum is shown for comparison. (d) Sublimed reference and OCVD films prepared from MgDPP and sublimed free-base porphyrins for comparison. (f) Sublimed reference and oCVD films prepared from AgDPP, and oCVD films from FeCIDPP for comparison. 
displayed signals at $398.5 \mathrm{eV}$ and $398.4 \mathrm{eV}$, respectively, corresponding to nitrogen in its initial $\mathrm{Zn}^{2+}$ and $\mathrm{Mg}^{2+}$ chemical environments. However, higher energy signals at $400.2 \mathrm{eV}$ and $400.3 \mathrm{eV}$ in pZnDPP and pMgDPP, respectively, confirm the presence of protonated nitrogen, and hence the presence of free-base porphyrin units in both films. Moreover, XPS analysis of the metal core level of pMgDPP films (Fig. S11a in the ESI $\dagger$ ) showed a significant shift of the $\mathrm{Mg}$ 2p-related signal, supporting the modification of the chemical environment of the porphyrin center. Such a shift is not observed in the pZnDPP films (Fig. S11b in the ESI $\dagger$ ) due to a less remarkable demetallation effect, consistent with the LDI-HRMS analysis. Demetallation in pZnDPP and pMgDPP is not surprising since $\mathrm{Zn}^{2+}$ and $\mathrm{Mg}^{2+}$ are known labile cations ${ }^{29}$ and the demetallation of the zinc(II) porphyrin was previously reported in the oCVD reaction of $\mathrm{ZnDMP} .^{20}$

Evidence of demetallation followed by significant remetalation of the porphyrin core was observed in the LDIHRMS spectra of the pAgDPP film that is dominated by the peaks related to fused iron porphyrin dimers $\left[(\text { FeDPP })_{2}-7 \mathrm{H}-\right.$ $\mathrm{Cl}]^{+}$(Fig. 3e). In consistency with this, the XPS analysis in the $\mathrm{N}$ 1s region of the signal corresponding to the PAgDPP oCVD film (Fig. 3f) shows a slight shift toward higher energies, i.e. to a value closer to the binding energy characteristic of the nitrogen environment in the pFeDPP film $(399.1 \mathrm{eV})$ confirming that demetallation and re-metalation occur at a significant degree during the oCVD reaction of AgDPP. In addition, the substantial incorporation of iron into the resulting film was evidenced in the elemental composition, from the XPS analysis of the Fe $2 p$ core level (Table S2 of the ESI $\dagger$ ).

Both LDI-HRMS and XPS analyses indicate different reactivities of ZnDPP, MgDPP and AgDPP in oCVD, according to different $\mathrm{C}-\mathrm{C}$ coupling $\left(k_{1}\right)$, demetallation $\left(k_{2}\right)$ and remetalation $\left(k_{3}\right)$ rates. As shown in Scheme 2a, the oCVD reaction of ZnDPP is characterized by a slow demetallation rate that is balanced by the low redox potential of ZnDPP allowing an efficient intermolecular oxidative $\mathrm{C}-\mathrm{C}$ coupling. As a result, it yields highly fused porphyrins with partially demetallated oligomers (Fig. 3a and b). Porphyrin units resulting from remetalation were detected by LDI-HRMS, yet with a slighter contribution compared to the formation of demetallated oligomers (Fig. S7a, ESI $\dagger$ ). Analogously, the lowest redox potential of the MgDPP monomer allows a fast reactivity toward intermolecular oxidative dehydrogenative coupling. However the high lability of $\mathrm{Mg}^{2+}$ ions in porphyrins ${ }^{26}$ further leads to yielding highly fused porphyrin oligomers containing free-base, magnesium and iron porphyrin units (Scheme 2b), e.g. $\left[(\mathrm{MgDPP})\left(\mathrm{H}_{2} \mathrm{DPP}\right)-9 \mathrm{H}-\mathrm{Cl}\right]^{+}$and $[(\mathrm{MgDPP})(\mathrm{FeDPP})-6 \mathrm{H}-\mathrm{Cl}]^{+}$ (Fig. S7d, ESI $\dagger$ ). The re-metalation of porphyrin cores during the oCVD reaction with iron(III) from the oxidant and its byproducts is likely promoted by the faster demetallation of MgDPP compared to ZnDPP. It is important to mention that the formation of both free-base (demetallation) and iron(III) porphyrins (re-metalation) with higher redox potentials than MgDPP (Table 1) jeopardizes the formation of longer-fused porphyrin chains.



Scheme 2 Representation of the competing mechanisms during the oCVD reaction of (a) ZnDPP, (b) MgDPP, and (c) AgDPP porphyrins and classification of their intermolecular $\mathrm{C}-\mathrm{C}$ coupling $\left(k_{1}\right)$, demetallation $\left(k_{2}\right.$, represented by grey arrows) and re-metalation ( $k_{3}$, represented by orange arrows) rates. Intramolecular cyclization and chlorination side reactions are shown representatively.

Conversely, a lower extension of the intermolecular coupling was evidenced by UV-Vis-NIR for the pPdDPP, pRuDPP, pFeDPP, $\mathbf{p H}_{2}$ DPP, and pAgDPP films (Fig. 1c). In the case of pPdDPP, pRuDPP, and pFeDPP, LDI-HRMS does not provide any insight that could explain such behavior. Nevertheless, both PdDPP and FeClDPP possess high redox potentials $\left(E_{1 / 2}\right)$ that likely contribute to less efficient dehydrogenative coupling rates. In addition, the SOMO of palladium(II) diphenyl porphyrin radical cations is known to possess an $a_{1 \mathrm{u}}$ symmetry that induces regioselectivity towards meso-beta linkage. ${ }^{30-32}$ Therefore, predominately doubly linked fused porphyrin tapes are formed and absorption remains moderate in the NIR region.

On the other hand, ruthenium can exhibit multiple oxidation states when chelated in a porphyrin macrocycle. ${ }^{33}$ The oxidation of the central metal cation may therefore compete with the oxidative coupling of the porphyrins. Additionally, ruthenium porphyrins easily form oxo complexes, ${ }^{33}$ which may also interfere with the formation of highly conjugated and longfused porphyrin chains. Di-nuclear ruthenium porphyrin oxo complexes, i.e. $\left[(\mathrm{RuDPP})_{2}+\mathrm{O}\right]^{+}$and $\left[(\mathrm{RuDPP})_{3}+2 \mathrm{O}\right]^{+}$, are notably observed in the LDI-HRMS spectra of the sublimed reference film sRuDPP (Fig. S7i, ESI $\dagger$ ).

In the case of $\mathbf{p H}_{\mathbf{2}} \mathbf{D P P}$, none of the oligomers detected by LDI-HRMS contained free-based porphyrin units (Fig. S7k, 
ESI $\dagger$ ). Specifically, all the porphyrin units constituting the oligomers seem chelated with iron originating from the oxidant. The importance of the metalation reaction is confirmed by the observation of the XPS N 1s core level spectrum where a single contribution is observed at $399.1 \mathrm{eV}$, the same as that corresponding to the nitrogen environment in pFeDPP films (Fig. S12, ESI $\dagger$ ). This indicates that the metalation of the porphyrin macrocycle is essential to form fused porphyrin oligomers in oCVD, even though the high redox potential $\left(E_{1 / 2}\right)$ of FeClDPP yields weak dehydrogenative coupling rates.

The reactivity of AgDPP in oCVD points to a fast rate of demetallation (forming $\mathrm{H}_{2} \mathrm{DPP}$ ) followed by iron(III) incorporation into the porphyrin core (Scheme 2c). Indeed, mostly ironbased oligomers are detected in the LDI-HRMS spectrum of pAgDPP (Fig. 3e and Fig. S7h, ESI $\dagger$ ), while a single contribution, corresponding to a metal-nitrogen environment shifted to higher binding energy compared to the sublimed AgDPP monomer, is observed in the XPS N 1s core level spectrum (Fig. 3f). In addition, AgDPP has a relatively high redox potential (compared to ZnDPP and MgDPP), and it is even higher for FeClDPP, making the oxidative $\mathrm{C}-\mathrm{C}$ coupling reaction once again difficult. As a result, most likely shorter oligomers will be formed during the oCVD of AgDPP, with a lower degree of fusing, in good agreement with the previous analysis of the UV-Vis-NIR spectra of both the as-deposited and dichloromethane-rinsed oCVD coatings.

oCVD reactivity versus optoelectronic properties of the fusedmetalloporphyrin coatings

The expansion of the $\pi$-conjugated system is known to increase the energy of the highest occupied molecular orbital (HOMO) and leads to a low excitation gap in directly fused porphyrin tapes. ${ }^{9}$ Consistently, the dehydrogenative coupling of metalloporphyrins by oCVD leads to a broadened and shifted valence band edge towards lower binding energies compared to the sublimed reference films, as shown in Fig. S13 of the ESI. $\dagger$ From the valence band region of the XPS spectra, the valence band maxima (VBM) of the metalloporphyrin-based films were estimated (Fig. S14 of the ESI $\dagger$ ). The values are summarized in Table S3 (ESI $\dagger$ ) and presented in Fig. 4a.

Interestingly, pCuDPP and pNiDPP thin films show the most significant reduction of their VBMs, with a decrease of $1.28 \mathrm{eV}$. This is consistent with the previous UV-Vis-NIR analysis evidencing the prevalence of highly conjugated fused porphyrin chains (likely containing a significant proportion of triply linked units). Although highly conjugated structures are suggested by the optical characterization, pCoDPP and pZnDPP thin films show a lower reduction of their VBMs. The shifts of the VBMs were even less prominent for pPtDPP, pPdDPP, pAgDPP, and pRuDPP thin films, as shown in Fig. 4a. This behavior suggests a lower efficiency of the intermolecular dehydrogenative coupling for these cations, in agreement with the UV-Vis-NIR analysis. A negligible VBM shift is observed for pMgDPP, pFeDPP, and $\mathbf{p H}_{\mathbf{2}}$ DPP thin films in respect of their reference sublimed films. Multiple behaviors can be responsible for these low VBM shifts upon oCVD processing: i.e. high


Fig. 4 (a) Valence band maxima (VBM) estimated from the XPS analysis of sublimed and OCVD processed films. (b) Histograms of the currents obtained from the C-AFM measurements of the OCVD films.

redox potentials $\left(E_{1 / 2}\right)$ for FeClDPP, PdDPP and PtDPP, a pronounced ability to form meso-beta linkages for PdDPP and RuDPP, oxidation of the central metal cation for CoDPP, demetallation of the porphyrin macrocycle for ZnDPP, AgDPP and MgDPP, and re-metalation with iron for $\mathrm{H}_{2} \mathrm{DPP}$, AgDPP, MgDPP and ZnDPP. Particularly, the valence band edge XPS spectra of $\mathbf{p H}_{\mathbf{2}} \mathbf{D P P}$, pMgDPP and pFeDPP coatings almost all overlap, confirming the demetallation and re-metalation with iron originating from the oxidant.

The intrinsic conductivity of the directly fused porphyrin CP thin films arises from the combination of multiple features including the conjugation degree and length of the fused porphyrin tapes, ${ }^{34}$ the oxidation state of the central metal cation, intramolecular cyclization, and the demetallation/remetalation of the porphyrin core. Indeed, demetallation and further re-metalation with $\mathrm{Fe}^{3+}$ from the oxidising agent might lead to the presence of axial ligands that hinder the $\pi-\pi$ stacking of the chains, and thus the conductivity. Moreover, the presence of $\mathrm{Fe}^{3+}$ as the metal center might also favor the electron trapping, also affecting the conductivity. The c-AFM measurements reveal the notable influence of the metal cation on the intrinsic conductivity of the fused porphyrin films, as shown in Fig. $4 \mathrm{~b}$. 
The negative impact of the demetallation and re-metalation of the porphyrin macrocycles is reflected on the moderate conductivity of the PZnDPP films supposedly composed of triply fused porphyrin chains but partly composed of FeClDPP units. Such behavior is even more pronounced for $\mathbf{p M g D P P}$ that exhibits the lowest conductivity despite the fair dehydrogenative coupling rate, due to the significant demetallation and re-metalation. As result, a similar electronic feature is observed for $\mathbf{p H}_{2}$ DPP, pMgDPP, and pFeDPP oCVD coatings, in agreement with the VBM estimated from the XPS analysis shown in Fig. 4a. It is worth noting that the lowest conductivity displayed for the $\mathbf{p H}_{2} \mathbf{D P P}$ and $\mathbf{p F e D P P}$ coatings is expected from the lower yield in the formation of extended fused porphyrin tapes and the presence of $\mathrm{Fe}^{3+}$ at the center of the porphyrin macrocycles. On the other hand, the excellent conductivity of the pCuDPP film is in good agreement with the UV-Vis-NIR and XPS characterization pointing to a high efficiency of the intermolecular coupling, no alteration of the porphyrin core and the highest shift of the VBM.

Surprisingly, the pRuDPP film also displays a high conductivity, despite the lower coupling efficiency previously suggested for this coating. This observation hints at the presence of large oligomers due to a higher conjugation length of single-fused units. Conversely, the pNiDPP thin film was less conductive than expected from the high VBM shift, pointing to a poor ratio of triple:double linkages, and possible axial ligands at the $\mathrm{Ni}^{2+}$ center, as suggested in previous reports with di-mesitylsubstituted porphyrins (DMPs). ${ }^{23}$

The careful assessment and understanding of the optoelectronic properties are key to further photo-electrocatalytic applications - including solar-assisted solar fuel production - with directly fused metalloporphyrin CP photoelectrodes processed by oCVD. Therefore, the optical band gaps $\left(E_{\mathrm{g}}\right)$ of the fused metalloporphyrin films were estimated from the absorbance spectra using the Tauc plots, as presented in Fig. S15 of the ESI, $\uparrow$ and summarized in Table S4 of the ESI. $\dagger$ All the conjugated metalloporphyrin coatings showed $E_{\mathrm{g}}$ values between $2.25 \mathrm{eV}$ and $2.69 \mathrm{eV}$, a suitable interval for effective photon absorption, with the possibility of generating a sufficient driving force toward photocatalytic solar fuel production. ${ }^{35}$ Furthermore, given the VBM previously estimated from the valence band edge region of the XPS (Fig. 4a), and the $E_{\mathrm{g}}$ calculated from the absorbance spectra, the energy band alignment for the fused-metalloporphyrin coatings chelating different metal cations was estimated, as presented in Fig. 5.

All the directly fused porphyrin thin films show adequate conduction band minimum (CBM) values to allow electron injection toward the hydrogen evolution reaction (HER). Moreover, the role of $\mathrm{Zn}, \mathrm{Cu}$ and Co species as catalytic-active centers boosting the HER efficiency in porphyrin-based systems ${ }^{36-40}$ has been reported, in addition to the earth-abundance and nontoxicity of these elements. Therefore, the oCVD coatings of fused-metalloporphyrins of $\mathrm{Zn}$ (II), $\mathrm{Cu}$ (II) and $\mathrm{Co}$ (II) are highly promising for the development of photo-electrocatalytic hydrogen production devices.

Indeed, the theoretical maximum photocurrent achievable for each conjugated metalloporphyrin film can be estimated



Fig. 5 Energy band diagram of the fused metalloporphyrin thin films (pMDPP, $\mathrm{M}=\mathrm{Zn}, \mathrm{Cu}, \mathrm{Co}, \mathrm{Mg}, \mathrm{Ni}, \mathrm{Pt}, \mathrm{Pd}, \mathrm{Ag}, \mathrm{Ru}, \mathrm{FeCl}$, and $\mathrm{H}_{2}$ ). The redox potentials for proton reduction and water oxidation reactions in acid media are presented as references.

from its corresponding absorbance spectrum as: $j_{\mathrm{abs}}=(e / h c)$ $\int \lambda \cdot I(\lambda) \lambda \cdot\left(1-10^{-A}\right) \mathrm{d} \lambda$, where $I(\lambda)$ is the spectral irradiance, $e$ is the elemental charge, $h$ is the Planck constant and $c$ is the speed of light. These values are summarized in Table S4 of the ESI. $\dagger$ The pCoDPP film shows the highest expected photocurrent value, ideally being able to reach up to $5.77 \mathrm{~mA} \mathrm{~cm}^{-2}$, which corresponds to $5.29 \%$ of solar-to-hydrogen efficiency (STH). However, the combination of metalloporphyrins with suitable band alignment into a heterostructured device, providing both enhanced light absorption and charge transfer, can lead to a considerable increase in the performance of photoelectrodes based on these materials. In addition, the coatings from pNiDPP, pPtDPP, and pPdDPP could also be suitable for hole injection toward the oxygen evolution reaction, due to the adequate VBM position, below the water oxidation potential. It is worth noting that $\mathrm{Ni}$ species are well-known active catalytic sites for the OER, ${ }^{41,42}$ which can be beneficial to improve the reaction kinetics at the metalloporphyrin-based photoelectrode surface.

Therefore, owing to their facile processability when operating from the gas-phase and their suitable optoelectronic properties - as well as the ability of the existing metal cations to act as active catalytic sites - fused-metalloporphyrin coatings processed by oCVD can emerge as a new generation of photoelectrode materials for solar-assisted fuel production.

\section{Conclusions}

We have investigated the impact of the central metal cation on the dehydrogenative coupling of metalloporphyrins and on the optoelectronic properties of the resulting conjugated metalloporphyrin-based photoelectrodes. It was found out that the nature of the chelated cation has a strong influence on the regioselectivity for the $\mathrm{C}-\mathrm{C}$ coupling, as well as on the 
occurrence of side reactions. In particular, the demetallation of the porphyrin core plays a significant role in the constitution of conjugated polymers, following different kinetics according to the nature of the metal cation. The impact of the reactivity during the oCVD was evidenced in the optical band gap, band alignment and conductivity analysis of each metalloporphyrin coating. These results pave the way for the investigation and further implementation of conjugated metalloporphyrin-based photoelectrodes for clean and efficient solar-assisted fuel production.

\section{Author contributions}

D.C.M: investigation, formal analysis, writing - original draft, and writing - review and editing; E.V., M. H. and G. F: investigation, formal analysis, and writing - review and editing; N. B: conceptualization, methodology, formal analysis, funding acquisition, and writing - review and editing.

\section{Conflicts of interest}

There are no conflicts to declare.

\section{Acknowledgements}

We gratefully acknowledge the financial support from the European Research Council (ERC) through the ERC Consolidator Grant project CLEANH2 (grant agreement no. 865985). B. Marcolini, R. Vaudemont, J. Guillot, C. Vergne and P. Grysan of LIST are acknowledged for the TGA, XPS and c-AFM measurements.

\section{References}

1 T. Bessho, S. M. Zakeeruddin, C.-Y. Yeh, E. W.-G. Diau and M. Grätzel, Angew. Chem., Int. Ed., 2010, 49, 6646-6649.

2 I. Obraztsov, W. Kutner and F. D’Souza, Sol. RRL, 2017, 1, 1600002.

3 V. Nikolaou, G. Charalambidis, K. Ladomenou, E. Nikoloudakis, C. Drivas, I. Vamvasakis, S. Panagiotakis, G. Landrou, E. Agapaki, C. Stangel, C. Henkel, J. Joseph, G. Armatas, M. Vasilopoulou, S. Kennou, D. M. Guldi and A. G. Coutsolelos, ChemSusChem, 2021, 14, 961-970.

4 D. Khusnutdinova, A. M. Beiler, B. L. Wadsworth, S. I. Jacob and G. F. Moore, Chem. Sci., 2017, 8, 253-259.

5 M. Joseph and S. Haridas, Int. J. Hydrogen Energy, 2020, 45, 11954-11975.

6 A. Aziz, A. R. Ruiz-Salvador, N. C. Hernández, S. Calero, S. Hamad and R. Grau-Crespo, J. Mater. Chem. A, 2017, 5, 11894-11904.

7 E. Tavakoli, A. Kakekhani, S. Kaviani, P. Tan, M. M. Ghaleni, M. A. Zaeem, A. M. Rappe and S. Nejati, J. Am. Chem. Soc., 2019, 141, 19560-19564.

8 J. Wang, L. Xu, T. Wang, S. Chen, Z. Jiang, R. Li, Y. Zhang and T. Peng, Adv. Funct. Mater., 2021, 31, 2009819.
9 A. Tsuda and A. Osuka, Science, 2001, 293, 79-82.

10 T. Ishizuka, Y. Saegusa, Y. Shiota, K. Ohtake, K. Yoshizawa and T. Kojima, Chem. Commun., 2013, 49, 5939-5941.

11 M. C. Scharber and N. S. Sariciftci, Adv. Mater. Technol., 2021, 2000857.

12 H. Jia, Z. Sun, D. Jiang and P. Du, Chem. Mater., 2015, 27, 4586-4593.

13 I. Hijazi, T. Bourgeteau, R. Cornut, A. Morozan, A. Filoramo, J. Leroy, V. Derycke, B. Jousselme and S. Campidelli, J. Am. Chem. Soc., 2014, 136, 6348-6354.

14 D. Khusnutdinova, B. L. Wadsworth, M. Flores, A. M. Beiler, E. A. Reyes Cruz, Y. Zenkov and G. F. Moore, ACS Catal., 2018, 8, 9888-9898.

15 A. M. Huerta-Flores, G. Bengasi, K. Baba and N. D. Boscher, ACS Appl. Energy Mater., 2020, 3, 9848-9855.

16 E. A. Reyes Cruz, D. Nishiori, B. L. Wadsworth, D. Khusnutdinova, T. Karcher, G. Landrot, B. Lassalle-Kaiser and G. F. Moore, ChemElectroChem, 2021, 8, 3614-3620.

17 G. Bengasi, K. Baba, G. Frache, J. Desport, P. Gratia, K. Heinze and N. D. Boscher, Angew. Chem., Int. Ed., 2019, 58, 2103-2108.

18 K. Baba, G. Bengasi, D. El Assad, P. Grysan, E. Lentzen, K. Heinze, G. Frache and N. D. Boscher, Eur. J. Org. Chem., 2019, 2368-2375.

19 M. Heydari Gharahcheshmeh and K. K. Gleason, Adv. Mater. Interfaces, 2019, 6, 1801564.

20 G. Bengasi, J. S. Desport, K. Baba, J. P. Cosas Fernandes, O. De Castro, K. Heinze and N. D. Boscher, RSC Adv., 2020, 10, 7048-7057.

21 T. Tanaka and A. Osuka, Chem. Soc. Rev., 2015, 44, 943-969.

22 G. Bengasi, K. Baba, O. Back, G. Frache, K. Heinze and N. D. Boscher, Chem. - Eur. J., 2019, 25, 8313-8320.

23 G. Bengasi, L. Quétu, K. Baba, A. Ost, J. P. Cosas Fernandes, P. Grysan, K. Heinze and N. D. Boscher, Eur. J. Inorg. Chem., 2020, 1938-1945.

24 G. Bengasi, R. Meunier-Prest, K. Baba, A. Kumar, A. L. Pellegrino, N. D. Boscher and M. Bouvet, Adv. Electron. Mater., 2020, 6, 2000812.

25 H. G. Roth, N. A. Romero and D. A. Nicewicz, Synlett, 2016, 714-723.

26 K. Baba, G. Bengasi, F. Loyer, J. P. C. Fernandes, D. El Assad, O. De Castro and N. D. Boscher, ACS Appl. Mater. Interfaces, 2020, 12, 37732-37740.

27 X. Ke, R. Kumar, M. Sankar and K. M. Kadish, Inorg. Chem., 2018, 57, 1490-1503.

28 K. Laba, M. Lapkowski, D. L. Officer, P. Wagner and P. Data, Electrochim. Acta, 2020, 330, 135140.

29 M. Speckbacher, L. Yu and J. S. Lindsey, Inorg. Chem., 2003, 42, 4322-4337.

30 Y. Nakamura, N. Aratani, A. Tsuda, A. Osuka, K. Furukawa and T. Kato, J. Porphyrins Phthalocyanines, 2003, 07, 264-269.

31 C.-M. Feng, Y.-Z. Zhu, S.-C. Zhang, Y. Zang and J.-Y. Zheng, Org. Biomol. Chem., 2015, 13, 2566-2569.

32 M. Kamo, A. Tsuda, Y. Nakamura, N. Aratani, K. Furukawa, T. Kato and A. Osuka, Org. Lett., 2003, 5, 2079-2082. 
33 J. P. Collman, C. E. Barnes, P. J. Brothers, T. J. Collins, T. Ozawa, J. C. Gallucci and J. A. Ibers, J. Am. Chem. Soc., 1984, 106, 5151-5163.

34 N. Algethami, H. Sadeghi, S. Sangtarash and C. J. Lambert, Nano Lett., 2018, 18, 4482-4486.

35 R. van de Krol, Y. Liang and J. Schoonman, J. Mater. Chem., 2008, 18, 2311-2320.

36 Y. Liu, Y. Han, Z. Zhang, W. Zhang, W. Lai, Y. Wang and R. Cao, Chem. Sci., 2019, 10, 2613-2622.

37 H. Lei, Y. Wang, Q. Zhang and R. Cao, J. Porphyrins Phthalocyanines, 2020, 24, 1361-1371.
38 S. Cui, M. Qian, X. Liu, Z. Sun and P. Du, ChemSusChem, 2016, 9, 2365-2373.

39 B. B. Beyene, S. B. Mane and C.-H. Hung, J. Electrochem. Soc., 2018, 165, H481-H487.

40 P. T. Smith, B. P. Benke, L. An, Y. Kim, K. Kim and C. J. Chang, ChemElectroChem, 2021, 8, 1653-1657.

41 V. Vij, S. Sultan, A. M. Harzandi, A. Meena, J. N. Tiwari, W.-G. Lee, T. Yoon and K. S. Kim, ACS Catal., 2017, 7, 7196-7225.

42 I. Elghamry, A. S. Alablan, M. A. Alkhalifah and M. E. Abdelsalam, J. Electrochem. Soc., 2021, 168, 016510. 